\title{
Gene Therapy of Pancreatic Cancer With Green Fluorescent Protein and Tumor Necrosis Factor-Related Apoptosis-Inducing Ligand Fusion Gene Expression Driven by a Human Telomerase Reverse Transcriptase Promoter
}

\author{
Matthew H. Katz, MD, Daniel E. Spivack, MD, Shinako Takimoto, BS, \\ Bingliang Fang, MD, PhD, Douglas W. Burton, MS, A. R. Moossa, MD, \\ Robert M. Hoffman, PhD, and Michael Bouvet, MD
}

\begin{abstract}
Background: Tumor necrosis factor-related apoptosis-inducing ligand (TRAIL) induces apoptosis in malignant cells but not in normal cells. Ad/g-TRAIL, an adenoviral vector in which expression of green fluorescent protein (GFP) and TRAIL is driven by a human telomerase reverse transcriptase promoter, has shown promise as a targeted antitumor agent.

Methods: To investigate the effects of TRAIL gene therapy on pancreatic cancer, BxPC-3, MIA-PaCa-2, Panc-1, and ASPC-1 cells were treated with Ad/g-TRAIL. Transfection and protein expression were determined by using immunoblotting and identification of GFP with fluorescent microscopy and flow cytometry. Cell viability was determined by proliferation assay. Cell-cycle analysis and quantification of caspase- 3 were used to identify apoptosis. The in vivo efficacy of Ad/g-TRAIL was characterized in a novel red fluorescent protein murine model of MIA-PaCa-2 pancreatic cancer.

Results: Cells treated with Ad/g-TRAIL expressed GFP and exhibited apoptotic morphology within 2 days of treatment. Treatment with this vector in vitro resulted in less cell viability, increased caspase- 3 activity, and a greater apoptotic fraction than treatment with controls. In vivo, treatment with Ad/g-TRAIL significantly suppressed tumor growth.

Conclusions: TRAIL gene therapy induces apoptosis of pancreatic tumor cells both in vitro and in vivo and is a promising therapy in the treatment of pancreatic cancer.
\end{abstract}

Key Words: Pancreatic cancer-Gene therapy-TRAIL_GFP-RFP.

Pancreatic ductal adenocarcinoma is one of the most lethal human malignancies, accounting for more than 30,000 deaths yearly in the United States alone. ${ }^{1}$ Treat-

\footnotetext{
Received January 27, 2003; accepted May 15, 2003.

From the Departments of Surgery (MHK, DS, ST, ARM, RMH, MB) and Medicine (DWB), University of California at San Diego, San Diego, California; Department of Cardiovascular and Thoracic Surgery (BF), University of Texas M. D. Anderson Cancer Center, Houston Texas; and AntiCancer, Inc. (RMH), San Diego, California.

Presented at the 56th Annual Meeting of the Society of Surgical Oncology, Los Angeles, CA, March 7, 2003.

Address correspondence and reprint requests to: Michael Bouvet, MD, Department of Surgery, University of California, San Diego, 3350 La Jolla Village Dr. (112E), San Diego, CA 92161; Fax: 858-552-4352; E-mail: mbouvet@ucsd.edu.

Published by Lippincott Williams \& Wilkins (C) 2003 The Society of Surgical Oncology, Inc.
}

ment of this disease has traditionally required aggressive multimodality therapy, including pancreaticoduodenectomy and adjuvant chemoradiation. Nonetheless, only $10 \%$ to $15 \%$ of these cancers are typically found to be resectable at the time of diagnosis. ${ }^{2}$ Moreover, treatment of these tumors with chemoradiation has been less successful than in other tumors, such as those of the rectum or breast. Accordingly, despite advances in these fundamental therapeutic techniques, patient survival with pancreatic cancer is grim, and the disease is almost uniformly fatal. Currently, even in the most experienced centers, patient median survival after resection remains less than 21 months. $^{3-5}$

Recently, investigation into alternative treatment strategies for this aggressive disease has led to advances in 
the field of gene therapy for pancreatic cancer. By using gene-therapy techniques, genes encoding proteins with antitumor effects are delivered with viral vectors into human cancers, with the potential for a reduction in tumor load. One such gene is tumor necrosis factorrelated apoptosis-inducing ligand (TRAIL/Apo-2L). ${ }^{6,7}$ This gene, which is constitutively expressed in many normal human tissues, encodes a type II transmembrane protein that has been shown to induce apoptosis in several human cancers, including colon and lung, through interaction with the DR4 and DR5 death receptors. ${ }^{8}$ The use of TRAIL in cancer therapy has seemed particularly attractive because its proapoptotic effects are lethal in transformed tumor cells but not in normal human cells. $^{9-11}$ Overexpression of TRAIL by using direct introduction of the gene through an adenoviral vector has been shown to induce apoptosis and suppress tumor growth both in vitro and in vivo in models of human lung and colon adenocarcinoma. ${ }^{12}$ Additionally, recombinant TRAIL protein has been demonstrated to have variable efficacy in the in vitro suppression of pancreatic cancer cell growth, depending on cell lineage. ${ }^{13}$

The use of gene-therapy techniques is limited by the systemic toxicity of both viral vectors and gene products. Even cancer-specific TRAIL activity has been reported to be associated with toxicity to other normal tissues in vivo, including brain ${ }^{14}$ and liver. ${ }^{15} \mathrm{~A}$ tumor-specific vector designed to target proapoptotic genes to cancers was therefore developed. ${ }^{16}$ This system uses a human telomerase reverse transcriptase (hTERT) ${ }^{17}$ promoter, which is active in most human cancer cell lines but inactive in normal somatic cells, ${ }^{18}$ to successfully induce tumorspecific transgene expression. Because it is a relatively weak promoter, however, the first-generation vector required transactivation by a GAL4/VP16 regulatory system, which significantly improved its efficiency. ${ }^{19}$ Subsequent modifications to the vector and the inclusion of the TRAIL gene resulted in Ad/g-TRAIL, a bicistronic vector that expresses two cassettes: one for a green fluorescent protein (GFP)-TRAIL fusion gene and one for a GAL4/VP16 regulatory component driven by the hTERT promoter. Use of this vector has been shown to effectively target TRAIL to certain cancers while inducing minimal toxicity in nontransformed cells. ${ }^{20}$

In this study, we examined the proapoptotic and antitumor effect of Ad/g-TRAIL on four different pancreatic cancer cell lines. In addition, we used a red fluorescent protein (RFP) in vivo model of pancreatic cancer to investigate the use of TRAIL-mediated gene therapy in the treatment of pancreatic ductal adenocarcinoma. Combined, our results demonstrate the ability of TRAIL gene therapy to effectively suppress the growth of pancreatic tumors.

\section{MATERIALS AND METHODS}

\section{Cell Lines}

Four different human pancreatic cancer cell linesBxPC-3, MIA-PaCa-2, Panc-1, and ASPC-1-were obtained from the American Type Culture Collection (Rockville, MD). Cells were maintained in media as recommended by the manufacturer: BxPC-3, Panc-1, and ASPC-1 cells were cultured in RPMI 1640 medium, whereas MIA-PaCa-2 cells were maintained in Dulbecco's modified Eagle medium. All cells were supplemented with $10 \%$ heat-inactivated fetal bovine serum and $1 \%$ penicillin and streptomycin (Gibco-BRL, Life Technologies, Inc., Grand Island, NY) and were incubated at $37^{\circ} \mathrm{C}$ in a $5 \% \mathrm{CO}_{2}$ incubator.

\section{RFP Retroviral Transduction and Selection of BxPC-3-RFP and MIA-PaCa-2-RFP Pancreatic Cancer Cells}

The pDsRed-2 vector (Clontech Laboratories Inc., Palo Alto, CA) was used to engineer BxPC-3 and MIA$\mathrm{PaCa}-2$ clones stably expressing RFP. This vector expresses RFP and a neomycin-resistance gene on the same bicistronic message. pDsRed-2 was produced in PT67 packaging cells, and then RFP transduction was initiated by incubating $20 \%$ confluent MIA-PaCa- 2 and BxPC-3 cells with retroviral supernatants of the packaging cells and Dulbecco's modified Eagle medium for 24 hours. Fresh medium was replenished at this time, and cells were allowed to grow in the absence of retrovirus for 12 hours. This procedure was repeated until high levels of RFP expression, as determined with fluorescence microscopy, were achieved. Cells were then harvested by trypsin/EDTA and subcultured into selective medium that contained $200 \mu \mathrm{g} / \mathrm{mL}$ of the aminoglycoside G418 (Geneticin; Invitrogen Corp., Carlsbad, CA). The level of G418 was increased to $2000 \mu \mathrm{g} / \mathrm{mL}$ stepwise. Clones expressing high levels of RFP were isolated with cloning cylinders as needed and were amplified and transferred by using conventional culture methods. High RFP-expression clones were isolated in the absence of G418 for 10 passages to select for stable expression of RFP in vivo.

\section{Recombinant Adenoviral Vectors}

The Ad/g-TRAIL and Ad/cytomegalovirus (CMV)GFP vectors were constructed as described previously. ${ }^{20}$ Ad/g-TRAIL is a bicistronic vector containing expression cassettes for both a GFP-TRAIL fusion protein 
driven by a synthetic GT promoter composed of five sets of GAL4 binding sites and a TATAA sequence and a Gal4/VP16 transactivator driven by the hTERT promoter. Viral titers were determined by optical absorbance at $A_{260} \mathrm{~nm}\left(1 \mathrm{~A}_{260} \mathrm{~nm}\right.$ unit $=10^{12}$ particles per milliliter) and by a plaque-forming assay. Titers determined by $\mathrm{A}_{260} \mathrm{~nm}$ measurement were used in all of the experiments. All viral preparations were determined to be free of contamination with $\mathrm{E}^{+}$adenovirus and endotoxin.

\section{Determination of TRAIL Overexpression by Western Blot}

Cultures of BxPC-3 cells were transduced with Ad/gTRAIL or Ad/CMV-GFP at a multiplicity of infection (MOI) of 2000 viral particles per cell for 48 hours. Whole cell extracts were prepared in chilled lysis buffer (50 mM of Tris-HCL, $150 \mathrm{mM}$ of $\mathrm{NaCl}, 1 \% \mathrm{NP}-40,1$ $\mathrm{mM}$ of dithiothreitol, $.5 \mathrm{mM}$ of phenylmethylsulfonyl fluoride, $1 \mu \mathrm{g} / \mathrm{mL}$ of leupeptin, and $10 \mu \mathrm{g} / \mathrm{mL}$ of aprotinin). Cell debris was then removed by centrifugation, and the supernatants were stored at $-80^{\circ} \mathrm{C}$. Protein concentration of the cell lysate was determined by using a BCA Protein Assay kit (Pierce, Rockford, IL). Samples were prepared by mixing aliquots of the protein extracts with NuPAGE $4 \times$ sample buffer (Invitrogen) and 10\% $\beta$-mercaptoethanol. Heat-denatured protein samples were run on a NuPAGE $10 \%$ Bis-Tris gel at $150 \mathrm{~V}$ for 1 hour and electrotransferred to a polyvinylidene fluoride membrane (Millipore Co., Billerica, MA). The membrane was blocked in 5\% nonfat dry milk/.1\% Trisbuffered saline-Tween solution for 1 hour at $37^{\circ} \mathrm{C}$. The membrane was then incubated with a 1/200 dilution of TRAIL rabbit polyclonal antibody (Santa Cruz Biotechnology, Santa Cruz, CA) and then with a secondary antibody consisting of horseradish-peroxidase-linked anti-rabbit immunoglobulin $G$ antibody (1/2500 dilution). Anti- $\beta$-actin antibody (1/5000; Sigma Chemical Co., St. Louis, MO) was used to confirm equal protein loading. Proteins were visualized by enhanced chemiluminescence (Supersignal West Pico Chemiluminescent Substrate; Pierce).

\section{Cell Viability Assay}

BxPC-3, MIA-PaCa-2, Panc-1, and ASPC-1 cells were distributed into 96-well plates at a density of 2000 cells per well 4 hours before virus infection. Cells were then treated with Ad/g-TRAIL or Ad/CMV-GFP at various MOIs, with phosphate-buffered saline (PBS) as a control treatment. The number of viable cells was subsequently determined with the CellTiter 96 Aqueous One Solution Cell Proliferation assay (Promega Corp., Mad- ison, WI) at 24-, 48-, 72-, 96-, and 120-hour time points after transfection. Briefly, at each time point, $20 \mu \mathrm{L}$ of CellTiter 96 solution was added to each well. The plates were then incubated for 1 hour, after which the absorbance of each well was read at a wavelength of $490 \mathrm{~nm}$. All assays were performed in quadruplicate, and each assay was repeated at least twice; results of representative experiments are shown as the mean of quadruplicate wells \pm SD.

\section{Fluorescence Microscopy}

BxPC-3, MIA-PaCa-2, Panc-1, ASPC-1, BxPC-3RFP, and MIA-PaCa-2-RFP cells were distributed into 96-well plates at a density of 2000 cells per well 4 hours before virus infection. Cells were treated with $\mathrm{Ad} / \mathrm{g}$ TRAIL, Ad/CMV-GFP, or PBS at MOIs determined to adversely affect cell viability on cell proliferation assay: 2000 viral particles per cell for BxPC-3, MIA-PaCa-2, and Panc-1 and 10,000 viral particles per cell for ASPC-1. Cells were then visualized and photographed after 72 hours of incubation by using a Nikon Eclipse TE300 inverted microscope (Nikon, Melville, NY) and were analyzed with MetaMorph imaging software (Universal Imaging Corp., Downington, PA).

\section{Flow Cytometric Assay}

BxPC-3 cells were plated into $100-\mathrm{mm}$ plates at a density of $1 \times 10^{6}$ cells per plate 4 hours before virus infection. Cells were then infected with Ad/g-TRAIL, Ad/CMV-GFP, or PBS by using a viral MOI of 2000 particles per cell. After incubation for 36 hours, both adherent and floating cells were harvested by trypsinization and washed with PBS. To quantify the sub- $\mathrm{G}_{1}$ fraction, cells were resuspended in 50\% ethanol and stained with $50 \mu \mathrm{g} / \mathrm{mL}$ of propidium iodide. The fraction of cells expressing GFP was assessed by fixing the cells in $2 \%$ paraformaldehyde. Cells were quantified by flow cytometric analysis performed in the flow cytometry core laboratory at our affiliated institution (Veterans Affairs Medical Center of San Diego, San Diego, CA) by using a Coulter (Miami, FL) Elite flow cytometer. The experiment was repeated twice with similar results.

\section{Caspase-3 Activity Assay}

BxPC- 3 cells were plated at a density of $1 \times 10^{6}$ cells per $150-\mathrm{mm}$ plate 4 hours before virus infection. Cells were then infected with Ad/g-TRAIL or Ad/CMV-GFP vectors at an MOI of 2000 particles per cell, by using PBS as a control treatment. After incubation for 48 hours, both adherent and floating cells were harvested by trypsinization. Caspase-3 activity was then determined by spectrophotometric assay with a commercially avail- 
able kit (Clontech). Briefly, $2 \times 10^{6}$ cells were incubated with a caspase- 3 substrate, DEVD-pNA, in the presence of dithiothreitol for 60 minutes at $37^{\circ} \mathrm{C}$. Relative caspase-3 activity was determined by spectrophotometric measurement of cleaved chromophore pNA, a caspase cleavage product, at $405 \mathrm{~nm}$. The correlation between protease activity and signal detection was confirmed by performing an additional control reaction with both $\mathrm{Ad} /$ g-TRAIL and a caspase-3 inhibitor. Each reaction was performed in triplicate, and the experiment was repeated with similar results.

\section{In Vivo Assessment of Ad/g-TRAIL on Pancreatic Cancer Cells}

Animal experiments were performed in accordance with the Guide for the Care and Use of Laboratory Animals (National Institutes of Health publication 85-23) under assurance number A3873-01. Human pancreatic cancer xenografts were established in 6- to 8-week-old female nude mice by the subcutaneous implantation of tumor into each mouse. The MIA-PaCa-2-RFP cell line was used for this purpose. This particularly aggressive cell line yields fast-growing, highly malignant tumors that are brightly fluorescent, permitting serial, high-resolution imaging of tumor growth.

Briefly, MIA-PaCa-2-RFP tumors in the exponential growth phase, grown subcutaneously in nude mice, were resected aseptically. Necrotic tissues were cut away, and the remaining healthy tumor tissues were cut with scissors and minced into $1-\mathrm{mm}^{3}$ pieces in RPMI 1640 medium. The twenty-four mice used in this study were then anesthetized, and each abdomen was sterilized with alcohol. A skin incision was then created in the right flank of each mouse, a subcutaneous pocket was created, and two tumor pieces were implanted. Once the tumors reached $.5 \mathrm{~cm}$ in size, as measured with calipers, the mice were randomized into treatment groups of eight mice each, and equivalent tumor size distribution in each group was confirmed. In situ, (1) intratumoral injection with adenoviral vector Ad/g-TRAIL or Ad/CMV-GFP at a dose of $6 \times 10^{10}$ viral particles per tumor in $100 \mu \mathrm{L}$ of PBS or (2) PBS sham treatment was then performed and was repeated 7 days later. Tumor volume was measured and calculated three times per week by blinded observers as volume $=a \times b^{2} / 2$, where $a$ is the largest diameter and $b$ is the smallest diameter. Additionally, tumor imaging was performed twice per week by placing each mouse in a fluorescent light box equipped with a fiberoptic light source of $470 \mathrm{~nm}$ (Lightools Research, Encinitas, CA) and capturing images on a charge-coupled device video camera.

\section{In Vivo Tumor Apoptosis Assay}

Apoptosis in explanted MIA-PaCa-RFP tumors was visualized by using an in situ terminal transferase-mediated dUTP nick end labeling (TUNEL) assay. Three mice underwent subcutaneous implantation of MIA$\mathrm{PaCa}-2-\mathrm{RFP}$ tumors as detailed previously and were treated with a single intratumoral injection of $\mathrm{Ad} / \mathrm{g}$ TRAIL or Ad/CMV-GFP at a dose of $6 \times 10^{10}$ viral particles per tumor in $100 \mu \mathrm{L}$ of PBS or were treated with PBS control when the tumors reached $.5 \mathrm{~cm}$ in size. Tumors were explanted 2 days after receiving this dose and were immediately placed in formalin. They were subsequently dehydrated in alcohol and embedded into paraffin. Tumors were then sectioned and mounted on glass slides. The TUNEL assay was performed with a commercial in situ apoptosis detection kit (ApopTag Peroxidase In Situ Apoptosis Detection Kit; Serologicals Corp, Norcross, GA). Briefly, rehydrated tissue samples were incubated with terminal deoxynucleotidyl transferase enzyme to end-label DNA fragments produced during apoptosis with digoxigenin. Samples were subsequently incubated with anti-digoxigenin peroxidase antibody and stained. Methyl green was used as a counterstain. Apoptotic cells were readily visible with brightfield microscopy.

To quantify and compare the rates of apoptosis between treatment groups, a semiquantitative scoring method was used. For each sample, the number of TUNEL-positive cells was counted per $400 \times$ highpower field (HPF). At least seven representative fields were evaluated by two blinded observers for each treatment group, from which an average value was calculated.

\section{Statistical Analysis}

Differences among treatment groups were assessed by using Student's $t$-test or analysis of variance with Statistica (StatSoft Inc., Tulsa, OK).

\section{RESULTS}

\section{Transgene Expression of Ad/g-TRAIL in Cultured Pancreatic Cell Lines}

Overexpression of the GFP-TRAIL fusion protein by pancreatic cancer cells in vitro after transfection by $\mathrm{Ad} /$ g-TRAIL was documented by Western blot (Fig. 1). Confirmation of successful transfection and protein expression was provided with fluorescence microscopy. After infection with Ad-g/TRAIL, which expresses the GFP-TRAIL fusion gene, all four lines of pancreatic cancer cells exhibited morphological changes consistent with cell death, including shrinkage, fragmentation, and 


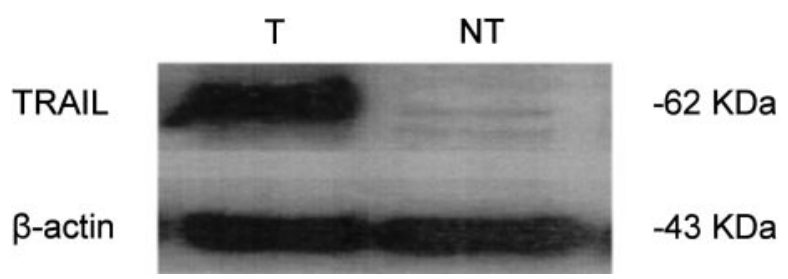

FIG. 1. Western blot analysis of BxPC-3 cells confirms green fluorescent protein/tumor necrosis factor-related apoptosis-inducing ligand (TRAIL) protein overexpression after transfection by Ad/g-TRAIL. Overexpression was visualized by using an antibody for TRAIL. T, BxPC-3 + Ad/g-TRAIL; NT, BxPC-3 + phosphate-buffered saline. The protein load was demonstrated by immunostaining for $\beta$-actin.

release from the monolayer, at the 72-hour time point (Fig. 2, top). The use of stable transductants of BxPC-3 and MIA-PaCa-2 cells engineered to stably express RFP facilitated clear identification of cells overexpressing the GFP-TRAIL fusion gene. These cells exhibited both green autofluorescence and apoptotic morphology (Fig. 2 , bottom), in contrast to cells that did not overexpress the fusion gene, which maintained their normal shape and red autofluorescence. In all cell lines, transfection with Ad/CMV-GFP resulted in high expression of GFP but minimal evidence of cell death. Significant GFP expression after treatment with both Ad/g-TRAIL and Ad/CMV-GFP was confirmed with fluorescence-activated cell-sorter analysis $(49.2 \%$ and $33.3 \%$, respectively; Fig. 3). As expected, no evidence of GFP or TRAIL expression was seen in cells controlled with PBS by using any of these modalities.

\section{TRAIL-Mediated Loss of Viability in Cultured Pancreatic Cell Lines}

To quantify the antitumor affect of TRAIL on the growth of pancreatic cancer cells in vitro, each pancreatic cancer cell line was treated with Ad/g-TRAIL and was subsequently analyzed with a cell proliferation assay. Viable cell proliferation was assessed at 24-hour intervals for 5 days after treatment. In each cell line, treatment with Ad/g-TRAIL led to a significant loss of viability as compared with treatment with either $\mathrm{Ad} /$ CMV-GFP or PBS, which became evident as early as 2 days after transfection (Fig. 4; $P<.05$ on days 3, 4, and 5 for all four cell lines). Growth suppression by TRAIL was achieved at MOIs of 2000 for BxPC-3, MIA$\mathrm{PaCa}-2$, and Panc-1 and at an MOI of 10,000 for ASPC-1.

\section{Proapoptosis Effect of TRAIL on Pancreas Cancer Cells In Vitro}

To characterize the growth-suppressive effects of TRAIL on pancreatic cancer cells, BxPC-3 cells were treated with Ad/g-TRAIL, and apoptosis was investigated by using fluorescence-activated cell-sorter analysis and by quantifying caspase- 3 activity. Cells transfected with the TRAIL-expressing vector had a dramatically higher sub- $\mathrm{G}_{1}$ fraction $(41.0 \%)$ than cells treated with either Ad/CMV-GFP or PBS (4.6\% and 6.9\%, respectively; Fig. 5). Similarly, apoptosis was clearly observed by measuring the activity of caspase-3, a key down-
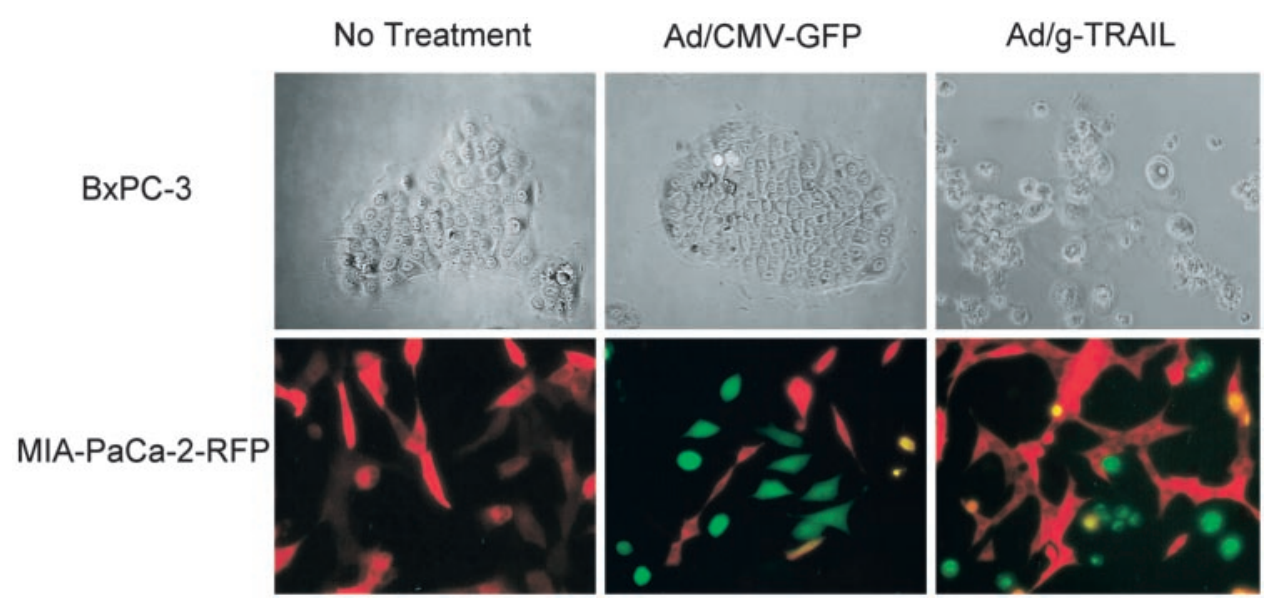

FIG. 2. Phase contrast and fluorescent microscopy of cell lines after treatment. Top panels: morphological changes consistent with apoptosis were seen after transfection of BxPC-3 human pancreatic cancer cells with Ad/g-tumor necrosis factor-related apoptosis-inducing ligand (TRAIL), but not Ad/cytomegalovirus (CMV)-green fluorescent protein (GFP). Similar results were observed in cell lines MIA-PaCa-2, ASPC-1, and Panc-1. Bottom panels: the transfection of MIA-PaCa-2 cells engineered to express red fluorescent protein (RFP) clearly identified cells overexpressing the GFP/TRAIL fusion gene, which both fluoresced green and exhibited apoptotic morphology. Cells that did not overexpress the fusion gene appeared normal in shape and maintained their innate red autofluorescence. Similar results were seen after transfection of RFP-expressing BxPC-3 cells. 

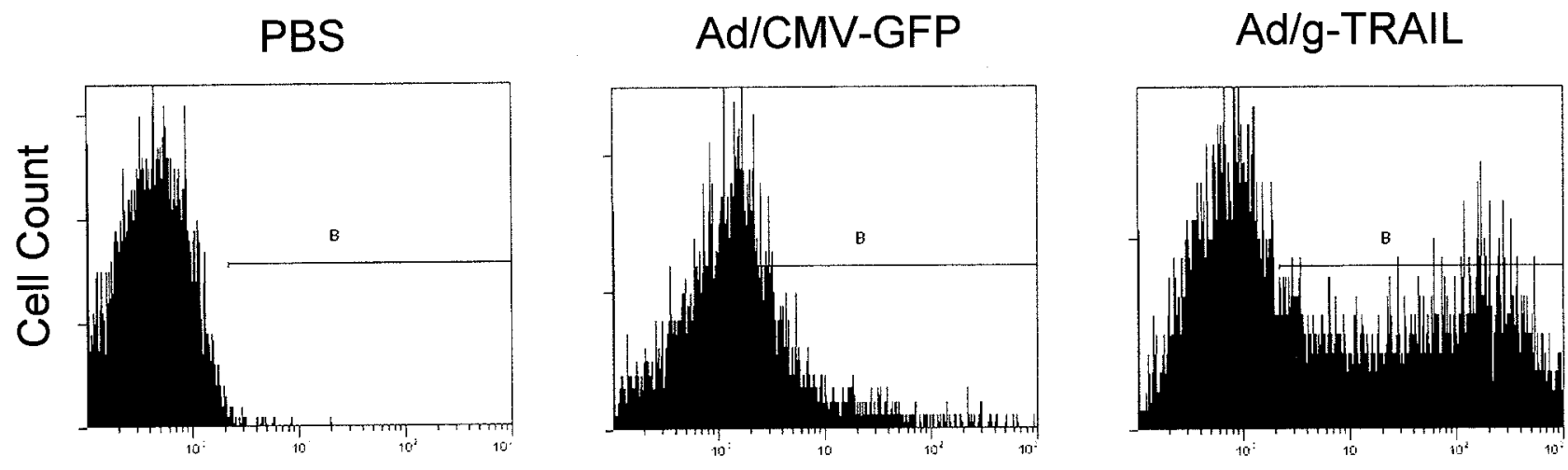

\section{Fluorescence Intensity}

FIG. 3. Levels of green fluorescent protein (GFP) expression were quantified by using flow cytometry. GFP was significantly expressed by BxPC-3 cells treated with both Ad/g-tumor necrosis factor-related apoptosis-inducing ligand (TRAIL; 49.2\%) and Ad/cytomegalovirus (CMV)-green fluorescent protein (GFP; 33.3\%), but not phosphate-buffered saline (PBS).

stream execution enzyme involved in apoptosis. A $>40$ fold increase in caspase- 3 activity in cells treated with Ad/g-TRAIL was observed compared with cells treated with vector or PBS control $(P<.05$; Fig. 6$)$. Caspase-3 activity was similar in cells treated with Ad/CMV-GFP and PBS $(P=.16)$.

\section{Effect of Ad/g-TRAIL on Pancreatic Cancer In Vivo}

To determine the efficacy of using gene therapy with Ad/g-TRAIL to treat pancreatic cancer in vivo, we used a subcutaneous, highly aggressive fluorescent xenograft model of MIA-PaCa-2 pancreatic tumors in nude mice.
BXPC-3

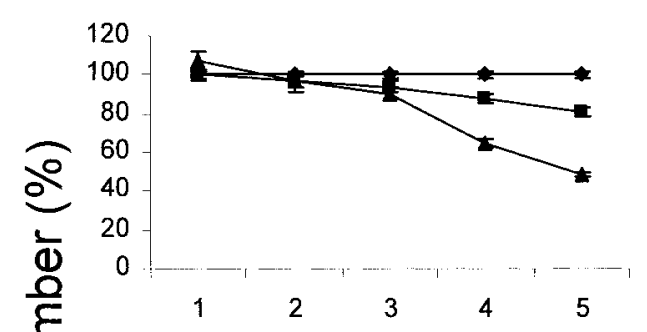

Panc-1

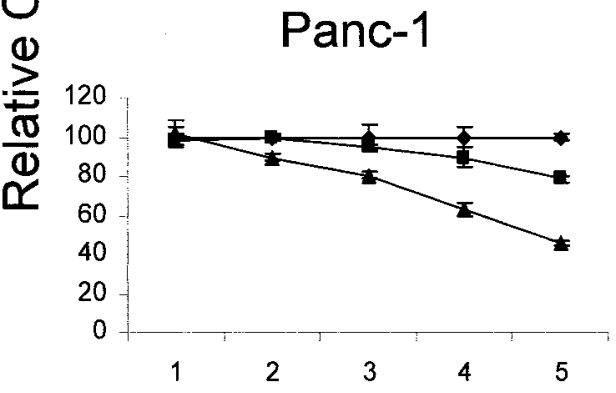

MIA-PaCa-2

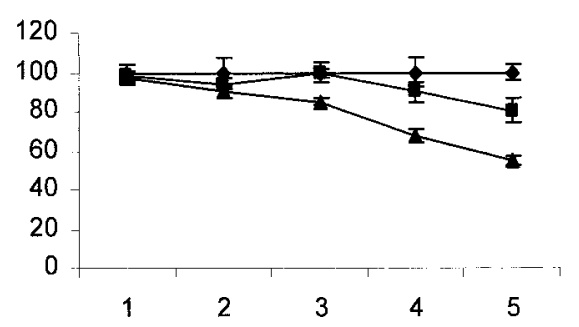

ASPC-1

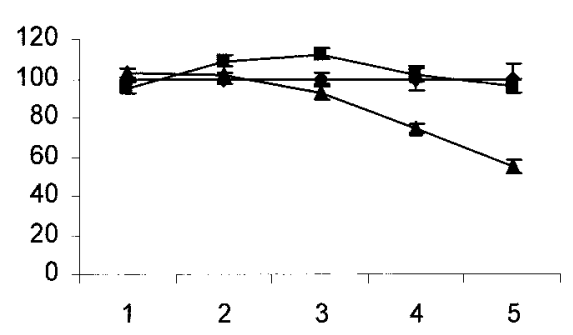

FIG. 4. In vitro effect of $\mathrm{Ad} / \mathrm{g}-$ tumor necrosis factor-related apoptosis-inducing ligand (TRAIL) on pancreatic cancer cell viability. The human pancreatic cancer cell lines BxPC-3, MIA-PaCa-2, Panc-1, and ASPC-1 were treated with $\mathrm{Ad} / \mathrm{g}$-TRAIL by using $\mathrm{Ad} /$ cytomegalovirus (CMV)-green fluorescent protein (GFP) as a vector control and phosphatebuffered saline (PBS) as a mock control. Ad/g-TRAIL significantly suppressed the growth of each cell line at varying multiplicities of infection: 2000 for BxPC-3, MIA-PaCa-2 and Panc-1, and 10,000 for ASPC-1 $(P<.05$ on days 3,4 , and 5 for all four cell lines). $\mathbf{A}, \mathrm{Ad} / \mathrm{g}-$ TRAIL; ם, Ad/CMV-GFP; PBS. Values represent the means \pm SD of quadruplicate wells.

Days After Treatment 

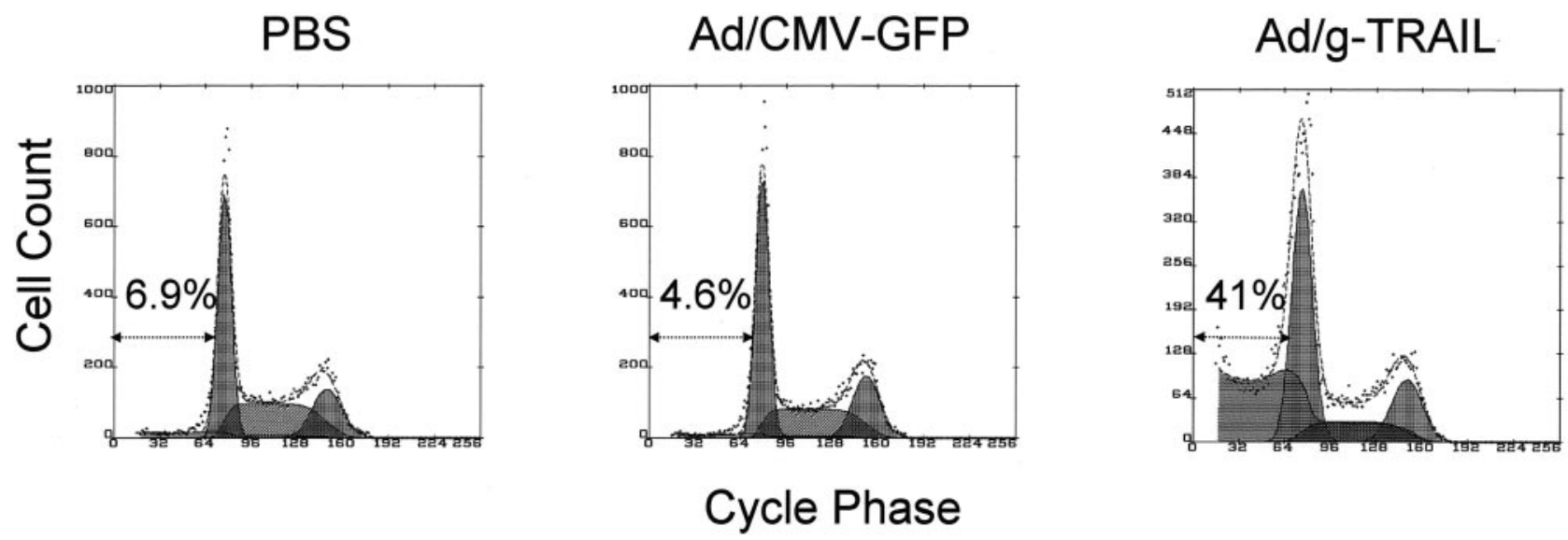

FIG. 5. The sub-G $\mathrm{G}_{1}$ fraction, determined by flow cytometry, of cells transfected with Ad/g-tumor necrosis factor-related apoptosis-inducing ligand (TRAIL; 41\%) was significantly larger than that of cells treated with either Ad/cytomegalovirus (CMV)-green fluorescent protein (GFP) or phosphate-buffered saline (PBS; $4.6 \%$ and $6.9 \%$, respectively).

These tumors were engineered to stably express RFP and were then subcutaneously implanted into treatment mice. Two intratumoral injections of $\mathrm{Ad} / \mathrm{g}$ TRAIL and Ad/CMV-GFP or mock treatment with PBS was then performed. In comparison with either control, treatment with Ad/g-TRAIL significantly suppressed tumor growth at each time point $(P \leq .05$; Fig. 7A). Tumors treated with Ad/CMV-GFP grew as fast as those treated with PBS. Stable expression of RFP by the pancreatic tumors facilitated dual-color in vivo fluorescent tumor imaging (Fig. 7B) and tumor measurement. Red tumors treated with Ad/CMV-GFP clearly demonstrated green fluorescence after intratumoral injection. Green fluorescence was not as apparent in tumors treated with Ad/g-TRAIL, although the hue of the tumor was somewhat altered after treatment. As expected, no green fluorescence was seen in tumors treated with PBS. It is important to note that no evidence of significant toxicity was noted in any of the treatment groups on physical examination.

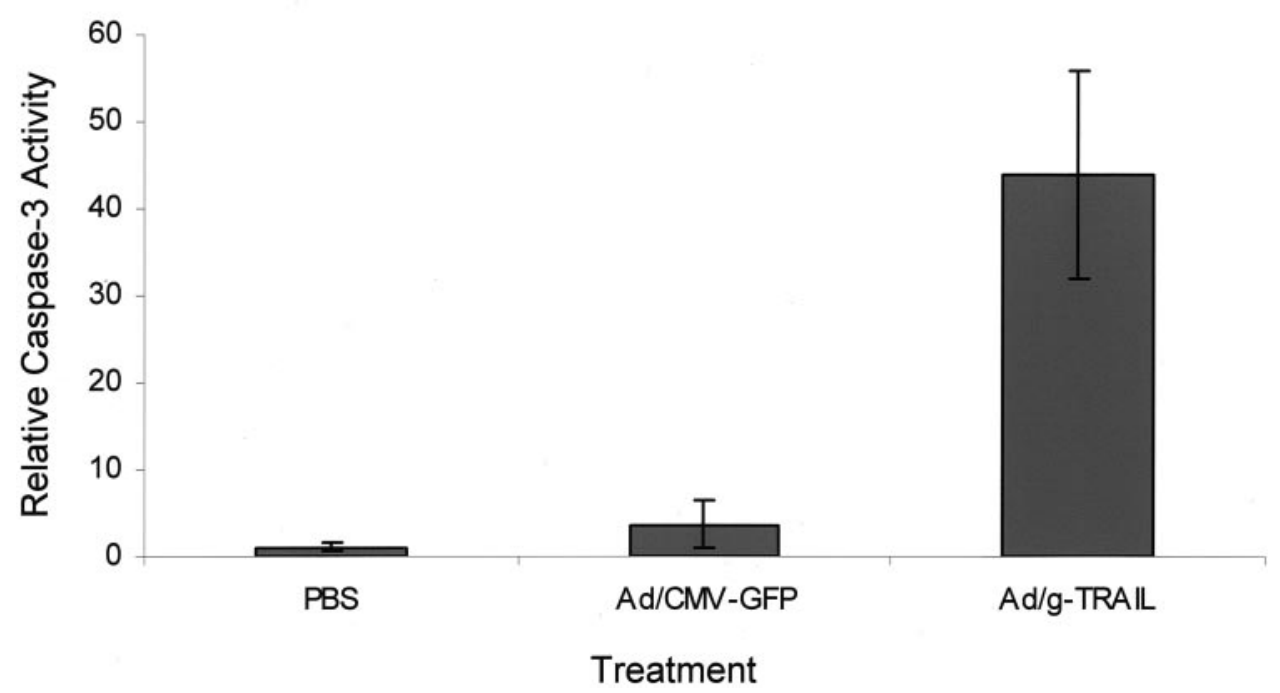

FIG. 6. Caspase-3 activity was increased after transfection of BxPC-3 cells with Ad/g-tumor necrosis factor-related apoptosis-inducing ligand (TRAIL). Caspase-3 is a cysteine protease that plays a key role in the execution phase of apoptosis. Values represent relative caspase-3 activity \pm SD 2 days after treatment with vectors or control. Caspase-3 levels were approximately 40 times greater in BxPC-3 cells after treatment with Ad/g-TRAIL compared with treatment with either Ad/cytomegalovirus (CMV)-green fluorescent protein (GFP) or phosphate-buffered saline (PBS; $P<.05)$. 
A

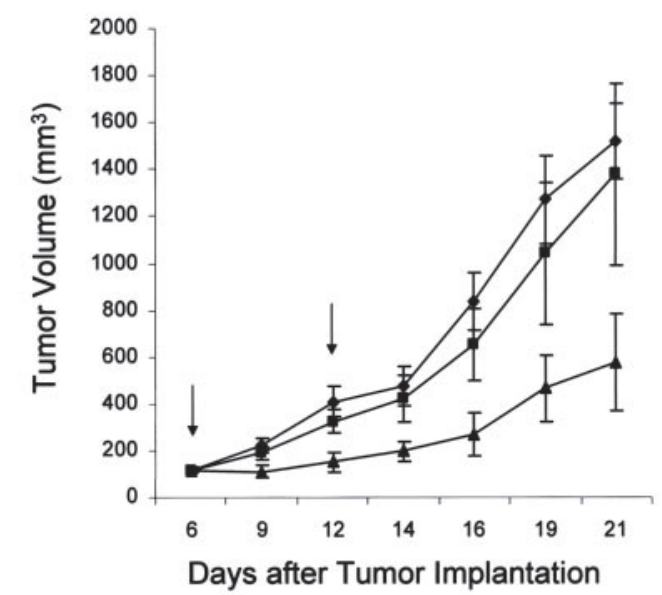

B
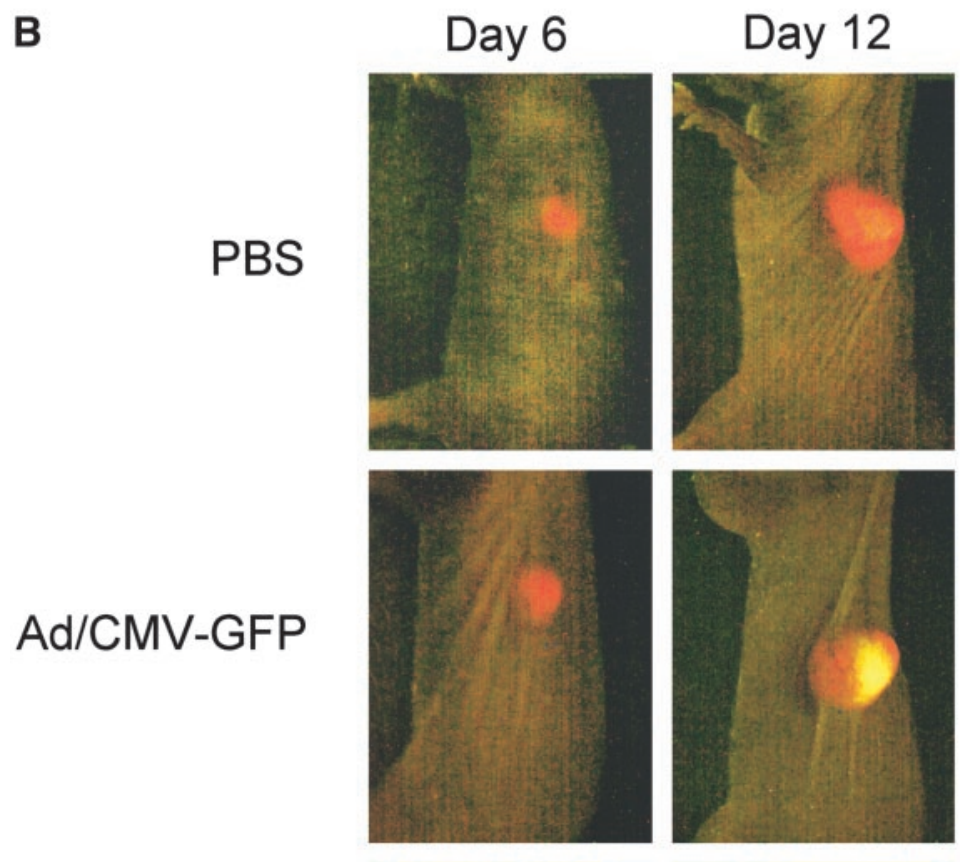

Day 20
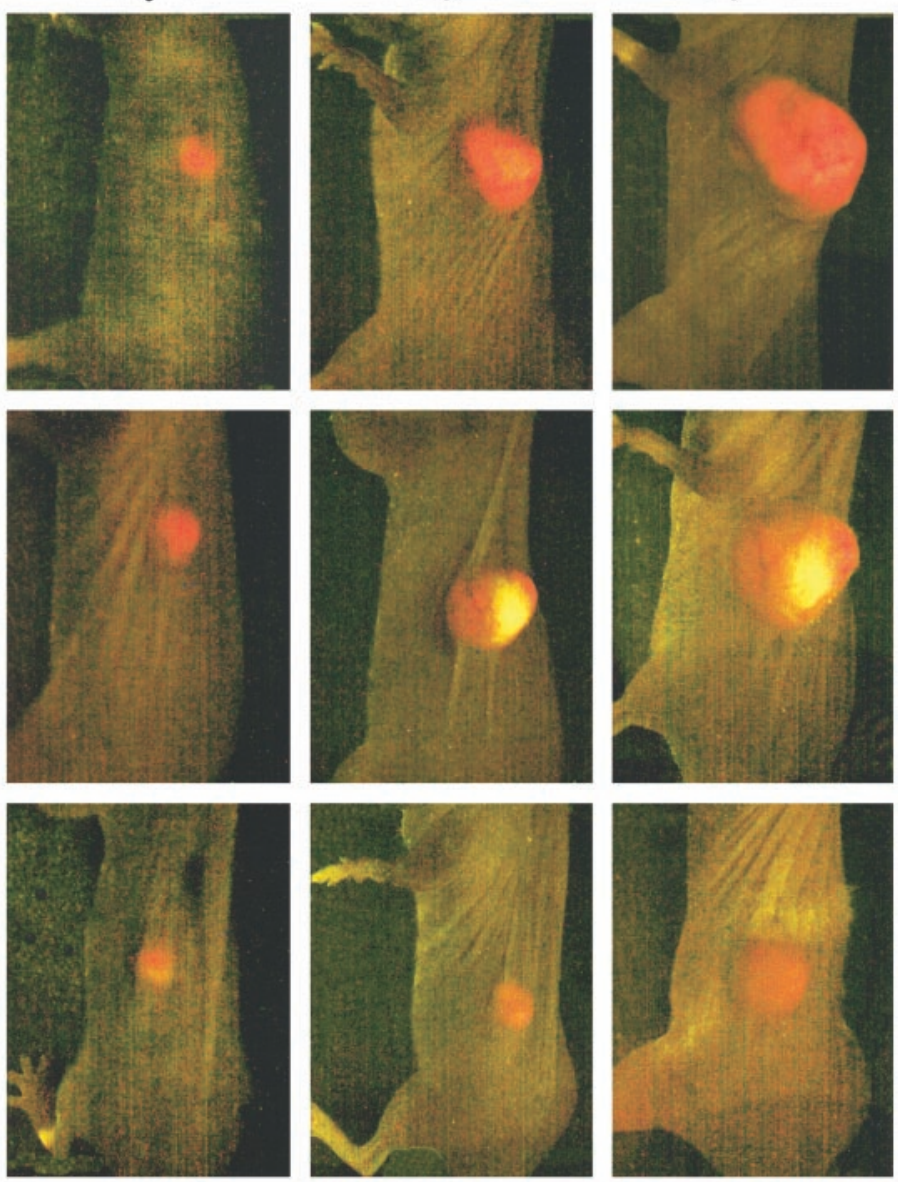

FIG. 7. Tumor growth in a xenograft mouse model of MIA-PaCa-2-red fluorescent protein (RFP) pancreatic cancer. (A) Tumor volume was monitored over time after subcutaneous tumor implantation. Arrows represent the time points at which vector treatment was administered. Values represent the mean tumor volume of 8 mice per treatment group. $\mathbf{\Delta}$, Ad/g-tumor necrosis factor-related apoptosis-inducing ligand (TRAIL); $\boldsymbol{\square}$, phosphate-buffered saline (PBS); $\bullet$ Ad/cytomegalovirus (CMV)-green fluorescent protein (GFP). Tumor growth was significantly suppressed in mice treated with Ad/g-TRAIL as compared with control mice ( $P \leq .05$ at each time point). (B) Direct, external in vivo imaging of MIA-PaCa-2-RFP tumor growth. Panels represent a representative mouse from each treatment group as imaged on day 6, 12, and 20 after tumor implantation. Tumors treated with both Ad/CMV-GFP and Ad/g-TRAIL exhibited green-on-red fluorescence in areas overexpressing their respective gene products, but the effect was far more dramatic after Ad/CMV-GFP administration. 
TUNEL staining of explanted tumors demonstrated a visibly higher level of apoptosis in tumors treated with Ad/g-TRAIL than controls (Fig. 8). Semiquantitative analysis of each sample confirmed a significantly higher level of apoptosis in tumors treated with TRAIL adenovirus (44 cells per HPF) compared with either vector control or PBS (17 and 15 cells per HPF, respectively; $P=.0002)$. The number of apoptotic cells did not differ between vector and mock controls $(P=.63)$.

\section{DISCUSSION}

The use of novel gene-therapy techniques in the treatment of pancreatic ductal adenocarcinoma has evolved over the past several years. Several strategies in gene therapy have been exploited, including restoring the wild-type forms of mutated genes through overexpression, introducing genes that promote a heightened susceptibility of malignant cells to other more conventional therapeutics, and inducing cell death through the transduction of proapoptotic genes. Using such methods, our laboratory and others have successfully induced pancreatic cancer cell death through the transduction of various genes, including wild-type $p 53^{21}$ and $p 16,,^{22}$ TIMP, ${ }^{23}$ and Bax. $^{24}$

The use of TRAIL in the therapy of pancreatic cancer seems promising, because it has been shown to have a significant antitumor effect in other solid human malignancies. First described in $1995,{ }^{6}$ the TRAIL protein is a cytokine in the tumor necrosis factor superfamily that triggers the external pathway of apoptosis through its interaction with the cellular death receptors DR4 and DR5.25 On ligand binding, adapter proteins such as FADD are recruited that bind and activate caspase-8, which in turn activates key downstream effectors of apoptosis such as caspase-3. This extrinsic pathway is linked to an intrinsic pathway of apoptosis that involves cytochrome $c$ release from mitochondria when triggered by molecules such as Bax, ${ }^{26}$ which subsequently activates the caspases.

The native TRAIL gene is found in many different normal human tissues and is primarily expressed as a
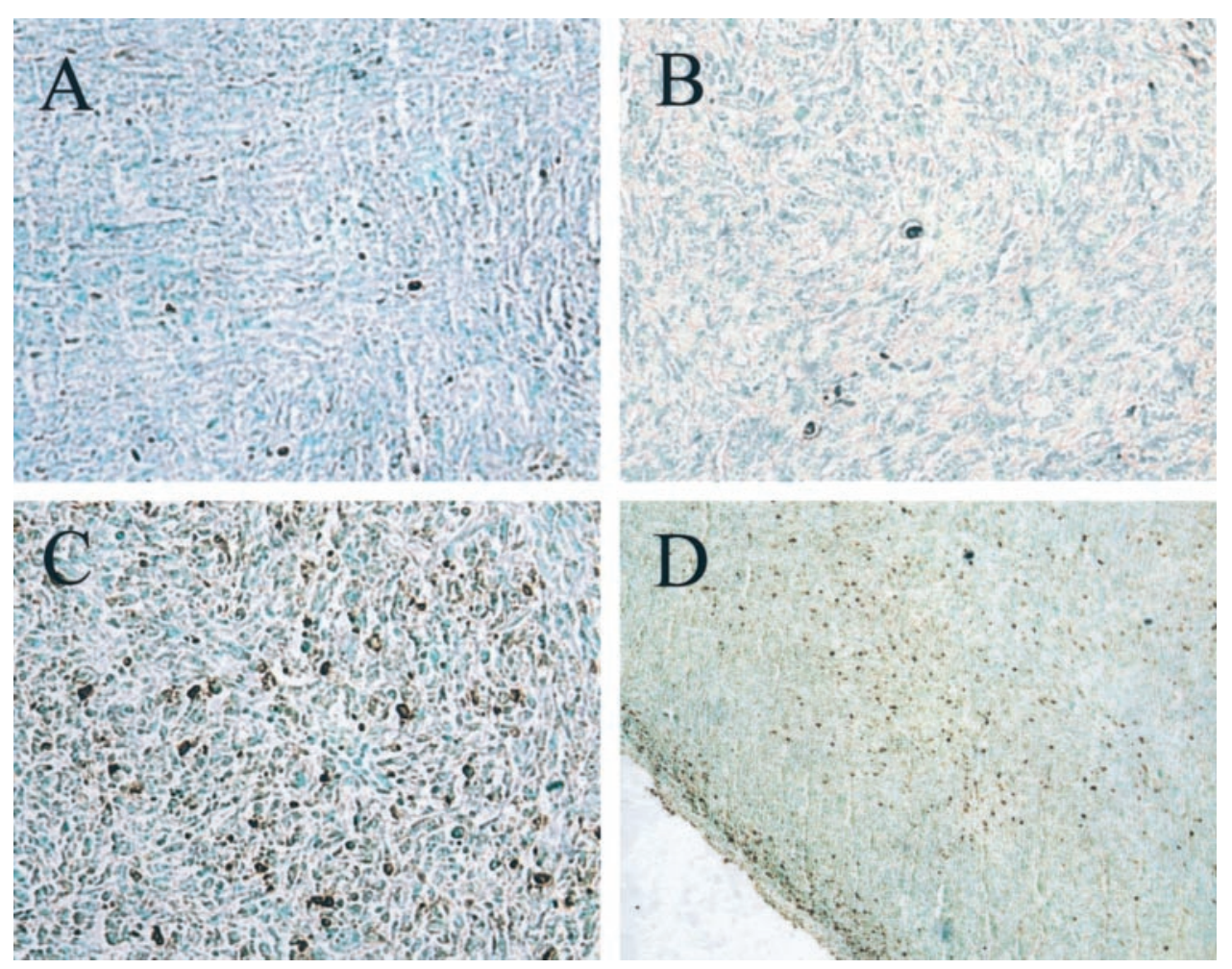

FIG. 8. Tumor necrosis factor-related apoptosis-inducing ligand (TRAIL) overexpression increased the percentage of terminal transferase-mediated dUTP nick end labeling-positive cells in in vivo MIA-PaCa-2-red fluorescent protein (RFP) pancreatic tumors. Tumors were explanted 2 days after treatment with Ad/g-TRAIL, Ad/cytomegalovirus (CMV)-green fluorescent protein (GFP), or phosphate-buffered saline (PBS), and apoptosis was detected in situ by using the immunohistochemical methods described in Materials and Methods. (A, B, C) Views ( $\times 400)$ of explanted MIA-PaCa-2-RFP tumors treated with PBS, Ad/CMV-GFP, and Ad/g-TRAIL, respectively. (D) View ( $\times 100)$ of an explanted MIA-PaCa-2-RFP tumor treated with Ad/g-TRAIL. Apoptotic cells, stained dark brown, were more abundant in tumors treated with Ad/g-TRAIL than in control treatments $(P=.0002)$. 
type II transmembrane protein approximately $34 \mathrm{kDa}$ in size. The extracellular region of the protein can be cleaved, forming a soluble TRAIL protein. Recombinant soluble TRAIL protein has been used in several studies examining the efficacy of TRAIL in suppressing the growth of cancers, including that of the pancreas. ${ }^{13}$ Significantly, membrane-bound TRAIL and soluble TRAIL have been shown to have different activities and ability to stimulate cellular death receptors: although soluble TRAIL seems to efficiently stimulate DR4, but not DR5, the membrane-bound protein is able to reliably stimulate both death receptors. ${ }^{13}$

Notably, the protein produced after cellular transfection with Ad/g-TRAIL has been shown to be the transmembrane, and not the soluble, form of TRAIL. ${ }^{12}$ This may explain the ability of therapy with Ad/g-TRAIL to effectively suppress tumor cell proliferation in the pancreatic cell line ASPC-1, which has previously been shown to be resistant to recombinant TRAIL chemotherapy. ${ }^{13,27}$ In our study, all four pancreatic tumor cell lines tested, including ASPC-1, demonstrated morphologic changes consistent with apoptosis and a significant loss of viability compared with untreated cells. A similar phenomenon has been demonstrated in the treatment of breast cancer cell lines, in which cell lines resistant to soluble TRAIL protein were susceptible to therapy with Ad/g-TRAIL. ${ }^{28}$ These results indicate that TRAIL-mediated gene therapy may be successful in the treatment of cancers that seem to be refractory to recombinant TRAIL treatment.

Our study did demonstrate a variable ability of TRAIL to induce cell death among several pancreatic cancer cell lines in vitro, confirming that TRAIL susceptibility is not uniform among pancreatic cancer types. In agreement with prior studies using recombinant TRAIL protein, we have shown BxPC-3 cells to be most sensitive to TRAILinduced cytotoxicity, followed by MIA-PaCa-2, PANC-1, and ASPC-1. Successful growth suppression of each cell line was achieved at MOIs within $1 \mathrm{log}$ of each other. Although the factors that determine the susceptibility of a pancreatic tumor line to TRAIL are not entirely clear, the presence or concentration of cellular death receptors does not seem to play a role. ${ }^{29}$

Nonetheless, certain preconditions for successful death receptor-mediated apoptosis have been identified. Deficiency in the expression of Bax, a key player in the intrinsic pathway of apoptosis, seems to confer resistance to TRAIL, ${ }^{30}$ whereas overexpression makes cells more sensitive to both TRAIL and other traditional chemotherapeutic agents, such as 5-fluorouracil and gemcitabine. ${ }^{31}$ In contrast, constitutive overexpression of bcl-2 has the opposite effect. One mechanism of relative TRAIL re- sistance therefore seems to involve the ratio of expression of these two genes by a particular cell line. ${ }^{26}$ It is unclear whether this mechanism, or others, is responsible for the relative resistance to TRAIL that we have identified in the ASPC-1 cell line.

A distinct advantage of gene therapy with $\mathrm{Ad} / \mathrm{g}$ TRAIL compared with therapy using recombinant TRAIL is that tumor-specific targeting is possible with this vector. Although the proapoptotic effect of TRAIL seems to be confined to malignant cells, TRAIL-associated toxicity has been reported in nontransformed tissue, including the liver and brain. Any such toxicity can be minimized by selectively targeting malignant cells and consequently selectively transfecting only these cells. This has been accomplished by driving the activation of TRAIL expression with the hTERT promoter. The hTERT gene is active in most primary cancers but is suppressed in normal human cells. ${ }^{18}$ Linking the expression of proapoptotic genes to this promoter has thus proven to limit protein expression and, thus, toxicity. ${ }^{16,20}$

It should be recognized that in this study, we used a novel, highly aggressive red fluorescent model of pancreatic cancer that uses MIA-PaCa- 2 cells engineered to stably express RFP. In this study, the model enabled clear visualization of GFP gene expression induced by adenoviral transfection with both Ad/g-TRAIL and Ad/ CMV-GFP, which appeared as a red to green color change. In vitro, this color change permitted definitive identification of populations of cells that overexpressed the GFP-TRAIL fusion gene after Ad/g-TRAIL transfection and confirmed that only cells that fluoresced green demonstrated apoptotic transformation, and vice versa. It also clearly revealed a population of cells that exhibited no evidence of GFP-TRAIL overexpression and, therefore, fluoresced red and exhibited normal cellular morphology. This population of cells is likely responsible for the continued, although decelerated, tumor growth seen after treatment with Ad/g-TRAIL and may explain our ability to induce pancreatic tumor growth suppression, but not tumor regression, with this agent.

In vivo, the red tumor autofluorescence facilitated sequential tumor imaging and adenovirus injection. In this setting, the green to red color change was less dramatic after transduction with Ad/g-TRAIL than Ad/ CMV-GFP, likely because the overexpression of the GFP-TRAIL fusion gene induces not only green fluorescence but also the transformation to apoptotic morphology. Ongoing studies involving intratumoral injection of TRAIL and other fluorescent-labeled gene therapy vectors into orthotopically implanted red fluorescent pancreatic tumors may demonstrate the benefits of this model, 
such as its ability to permit noninvasive image-guided injection of intra-abdominal tumors in the live animal.

We have demonstrated gene therapy with Ad/gTRAIL to effectively suppress pancreatic cancer growth both in vitro and in a novel, in vivo model of pancreatic cancer. We have also shown that therapy with Ad/gTRAIL may be superior to treatment of pancreatic cancer with recombinant TRAIL protein. Further studies are necessary and ongoing to characterize the ability of TRAIL gene therapy to control metastatic disease and improve long-term survival in patients with this devastating disease.

\section{ACKNOWLEDGMENTS}

The acknowledgments are available online at www.annalssurgicaloncology.org.

\section{REFERENCES}

1. Jemal A, Thomas A, Murray T, Thun M. Cancer statistics, 2002. CA Cancer J Clin 2002;52:23-47.

2. Sener SF, Fremgen A, Menck HR, Winchester DP. Pancreatic cancer: a report of treatment and survival trends for 100,313 patients diagnosed from 1985-1995, using the National Cancer Database. J Am Coll Surg 1999;189:1-7.

3. Bouvet M, Gamagami RA, Gilpin EA, et al. Factors influencing survival after resection for periampullary neoplasms. Am J Surg 2000;180:13-17.

4. Yeo CJ, Cameron JL, Sohn TA, et al. Six hundred fifty consecutive pancreaticoduodenectomies in the 1990s: pathology, complications, and outcomes. Ann Surg 1997;226:248-57; discussion 25760.

5. Breslin TM, Hess KR, Harbison DB, et al. Neoadjuvant chemoradiotherapy for adenocarcinoma of the pancreas: treatment variables and survival duration. Ann Surg Oncol 2001;8:123-32.

6. Wiley SR, Schooley K, Smolak PJ, et al. Identification and characterization of a new member of the TNF family that induces apoptosis. Immunity 1995;3:673-82.

7. Pitti RM, Marsters SA, Ruppert S, Donahue CJ, Moore A, Ashkenazi A. Induction of apoptosis by Apo-2 ligand, a new member of the tumor necrosis factor cytokine family. J Biol Chem 1996; 271:12687-90.

8. Pan G, O'Rourke K, Chinnaiyan AM, et al. The receptor for the cytotoxic ligand TRAIL. Science 1997;276:111-3.

9. French LE, Tschopp J. The TRAIL to selective tumor death. Nat Med 1999;5:146-7.

10. Walczak H, Miller RE, Ariail K, et al. Tumoricidal activity of tumor necrosis factor-related apoptosis- inducing ligand in vivo. Nat Med 1999;5:157-63.

11. Ashkenazi A, Pai RC, Fong S, et al. Safety and antitumor activity of recombinant soluble Apo2 ligand. J Clin Invest 1999;104:15562.

12. Kagawa S, He C, Gu J, et al. Antitumor activity and bystander effects of the tumor necrosis factor- related apoptosis-inducing ligand (TRAIL) gene. Cancer Res 2001;61:3330-8.

13. Ibrahim SM, Ringel J, Schmidt C, et al. Pancreatic adenocarci- noma cell lines show variable susceptibility to TRAIL-mediated cell death. Pancreas 2001;23:72-9.

14. Nitsch R, Bechmann I, Deisz RA, et al. Human brain-cell death induced by tumour-necrosis-factor-related apoptosis-inducing ligand (TRAIL). Lancet 2000;356:827-8.

15. Jo M, Kim TH, Seol DW, et al. Apoptosis induced in normal human hepatocytes by tumor necrosis factor-related apoptosisinducing ligand. Nat Med 2000;6:564-7.

16. Gu J, Kagawa S, Takakura M, et al. Tumor-specific transgene expression from the human telomerase reverse transcriptase promoter enables targeting of the therapeutic effects of the Bax gene to cancers. Cancer Res 2000;60:5359-64.

17. Bodnar AG, Ouellette M, Frolkis M, et al. Extension of life-span by introduction of telomerase into normal human cells. Science 1998;279:349-52.

18. Kim NW, Piatyszek MA, Prowse KR, et al. Specific association of human telomerase activity with immortal cells and cancer. Science 1994;266:2011-5.

19. Fang B, Ji L, Bouvet M, Roth JA. Evaluation of GAL4/TATA in vivo. Induction of transgene expression by adenovirally mediated gene codelivery. J Biol Chem 1998;273:4972-5.

20. Lin T, Gu J, Zhang L, et al. Targeted expression of green fluorescent protein/tumor necrosis factor- related apoptosis-inducing ligand fusion protein from human telomerase reverse transcriptase promoter elicits antitumor activity without toxic effects on primary human hepatocytes. Cancer Res 2002;62:3620-5.

21. Bouvet M, Bold RJ, Lee J, et al. Adenovirus-mediated wild-type p53 tumor suppressor gene therapy induces apoptosis and suppresses growth of human pancreatic cancer. Ann Surg Oncol 1998; 5:681-8.

22. Ghaneh P, Greenhalf W, Humphreys M, et al. Adenovirus-mediated transfer of p53 and p16(INK4a) results in pancreatic cancer regression in vitro and in vivo. Gene Ther 2001;8:199-208.

23. Bloomston M, Shafii A, Zervos EE, Rosemurgy AS. TIMP-1 overexpression in pancreatic cancer attenuates tumor growth, decreases implantation and metastasis, and inhibits angiogenesis. J Surg Res 2002;102:39-44.

24. Pirocanac EC, Nassirpour R, Yang M, et al. Bax-induction gene therapy of pancreatic cancer. J Surg Res 2002;106:346-51.

25. Ashkenazi A, Dixit VM. Death receptors: signaling and modulation. Science 1998;281:1305-8.

26. Roth W, Reed JC. Apoptosis and cancer: when BAX is TRAILing away. Nat Med 2002;8:216-8.

27. Matsuzaki H, Schmied BM, Ulrich A, et al. Combination of tumor necrosis factor-related apoptosis-inducing ligand (TRAIL) and actinomycin D induces apoptosis even in TRAIL-resistant human pancreatic cancer cells. Clin Cancer Res 2001;7:407-14.

28. Lin T, Huang X, Gu J, et al. Long-term tumor-free survival from treatment with the GFP-TRAIL fusion gene expressed from the hTERT promoter in breast cancer cells. Oncogene 2002;21:8020-8.

29. Satoh K, Kaneko K, Hirota M, Masamune A, Satoh A, Shimosegawa T. Tumor necrosis factor-related apoptosis-inducing ligand and its receptor expression and the pathway of apoptosis in human pancreatic cancer. Pancreas 2001;23:251-8.

30. LeBlanc H, Lawrence D, Varfolomeev E, et al. Tumor-cell resistance to death receptor-induced apoptosis through mutational inactivation of the proapoptotic Bcl-2 homolog Bax. Nat Med 2002; 8:274-81.

31. Xu ZW, Friess H, Buchler MW, Solioz M. Overexpression of Bax sensitizes human pancreatic cancer cells to apoptosis induced by chemotherapeutic agents. Cancer Chemother Pharmacol 2002;49: 504-10. 